\title{
OCORRÊNCIA DE DOIS CITÓTIPOS EM Serrasalmus spilopleura KNER, 1858 (CHARACIFORMES, SERRASALMIDAE) DA REGIÃO DE CONFLUÊNCIA DOS RIOS NEGRO E SOLIMÕES, AMAZONAS, BRASIL
}

\author{
Celeste Mutuko NAKAYAMA*, Jorge Ivan Rebelo PORTO*, Eliana FELDBERG*
}

RESUMO - Descreve-se pela primeira vez o cariótipo de exemplares de $S$, spilopleura da Amazônia Central. Todos os individuos analisados apresentaram número diplóide igual a 60 cromossomos, em conformidade com os dados da espécie obtidos em individuos da bacia do Paraná-Paraguai. No entanto, dois citótipos foram encontrados em simpatria na região de confluência dos rios Negro e Solimões, os quais não estão relacionados a heteromorfismo sexual. Além disso, os dois citótipos da Amazônia diferem dos três anteriormente descritos em exemplares do Paraná-Paraguai. A presença de distintos citótipos encontrados em simpatria e alopatria para $S$. spilopleura nos leva a sugerir que, ou essa espécie apresenta uma ampla heterogeneidade cariotipica ou existe um complexo de espécies cuja morfologia é muito semelhante.

Palavras-chave: citogenética, piranhas, Serrasalmidae, citótipos, bacia amazônica.

Occurrence of Two Cytotypes in Serrasalmus spilopleura Kner, 1858 (Characiformes, Serrasalmidae) from the Confluence Region of the Negro and Solimões Rivers, Amazonas, Brazil

ABSTRACT - The karyotype of Serrasalmus spilopleura from the Central Amazon is reported here for the first time. All analized individuals showed 60 chromosomes, as observed in previously published karyotypes of this species from the Paraná-Paraguai basin. However, two different cytotypes were found in sites near the confluence of the Negro and Solimões rivers, where the specimens were collected. Such karyotypic differences were not related to sex differentiation. Moreover, the two amazon cytotypes are different from the three cytotypes described in animals from Paraná-Paraguai basin. The different karyotypes detected so far, in both simpatry and allopatry conditions, suggest that $S$. spilopleura may be either a cytogenetically heterogeneous species or a complex of sibling species.

Key-words: cytogenetics, piranha, Serrasalmidae, cytotypes, Amazon basin.

\section{INTRODUÇÃO}

Serrasalmus spilopleura foi descrita em 1858 por Kner e é uma das espécies de piranha de mais ampla distribuição geográfica, ocorrendo nas bacias dos rios Solimões-Amazonas (Jégu, 1992), Tocantins-Araguaia (Jégu \& Santos, 1988), ParanáParaguai e Uruguai (Ringuelet et al., 1967; Géry et al., 1987) e habitando preferencialmente, a margem dos rios e lagos (Santos et al., 1984). É facilmente reconhecida por apresentar na nadadeira caudal uma faixa preta subterminal e uma faixa hialina terminal (Géry, 1977). Devido à sua coloração amarelada, é conhecida popularmente na Amazônia como piranha-tucupi. Porém, individuos adultos em periodo de reprodução geralmente perdem o tom amarelado e passam a apresentar a quase totalidade do corpo escurecida com tons prateados.

S. spilopleura já teve seu cariótipo descrito a partir de individuos coletados em diferentes

\footnotetext{
* Instituto Nacional de Pesquisas da Amazônia, Coordenação de Pesquisas em Biologia Aquática, Alameda Cosme Ferreira, 1756, 69.083-000, Manaus, AM. Brasil e-mail: feldberg@inpa.gov.br.
} 
localidades da bacia do ParanáParaguai (Galetti Jr. et al., 1985; Cestari \& Galetti Jr., 1992; MartinsSantos et al., 1994). Todos os indivíduos apresentaram número diplóide igual a 60 cromossomos e sistema múltiplo de regiões organizadoras de nucléolos (NORs) localizadas no braço curto dos cromossomos acrocêntricos. Contudo, diferenças estruturais nos cariótipos foram encontradas definindo três citótipos, sendo dois deles em simpatria (Cestari \& Galetti Jr., 1992).

No presente trabalho são descritos dois outros citótipos de $S$. spilopleura, detectados em indivíduos coletados na região de confluência dos rios Negro e Solimões, que são comparados com aqueles da bacia do Paraná-Paraguai.

\section{MATERIAL E MÉTODOS}

Os exemplares de Serrasalmus spilopleura (5 machos e 12 fêmeas) foram coletados na região de confluência dos rios Negro e Solimões entre o Catalão e a ilha da Marchantaria $\left(59^{\circ} 54^{\prime} \mathrm{W}, 3^{\circ} 09^{\prime} \mathrm{S}\right.$ e $60^{\circ} 00^{\prime} \mathrm{W}, 3^{\circ} 14^{\prime} \mathrm{S}$, respectivamente), próximo à cidade de Manaus.

Cromossomos metafásicos foram obtidos a partir de células de rim segundo as técnicas adaptadas para estudos cromossômicos de peixes por Bertollo et al. (1978) e Bertollo (1986). Os animais foram injetados, intraperitonealmente, com uma solução de fermento biológico e uma solução de colchicina $0,025 \%(1 \mathrm{ml} /$ $100 \mathrm{~g}$ de peso do animal), 24 horas e 45 minutos, antes de serem sacrificados respectivamente. Parte do rim foi retirada, fracionada numa solução hipotônica $(\mathrm{KCl}$ 0,075M) e fixada em fixador Carnoy (3 partes de metanol: 1 parte de ácido acético). A suspensão celular obtida foi pingada em lâminas de vidro, coradas com Giemsa $5 \%$ e examinadas em microscópio óptico.

Análises dos cromossomos foram realizadas em 647 células e o número diplóide foi determinado para machos e fêmeas. Os cromossomos foram medidos e arranjados em pares, em ordem decrescente de tamanho por grupos, ou seja, metacêntricos (M), submetacêntricos (SM), subtelocêntricos (ST) e acrocêntricos (A), considerando os limites propostos por Levan et al. (1964). O número fundamental ou número de braços foi determinado considerando os cromossomos metacêntricos, submetacêntricos e subtelocêntricos como tendo dois braços e os acrocêntricos como tendo um só braço.

\section{RESULTADOS}

Todos os 17 individuos de $S$. spilopleura analisados apresentaram número diplóide igual a 60 cromossomos.

Foram encontradas diferenças cromossômicas estruturais, não relacionadas com o sexo, que permitiram a identificação de duas fórmulas cariotípicas diferentes. Para 15 indivíduos (4 machos e 11 fêmeas), o cariótipo encontrado foi denominado citótipo "A", sendo constituído por $24 \mathrm{M}+20 \mathrm{SM}+4 \mathrm{ST}+$ $12 \mathrm{~A}$, perfazendo um número fundamental $\mathrm{NF}=108$ (Fig. 1A). Para 2 indivíduos ( 1 macho e 1 fêmea), o cariótipo encontrado foi denominado citótipo "B", sendo constituido por 
$24 \mathrm{M}+26 \mathrm{SM}+10 \mathrm{~A}$, perfazendo um número fundamental igual a 110 (Fig. 1B).
Na tabela 1 estão compilados os dados cariotípicos já obtidos para $S$. spilopleura.

Tabela 1. Compilação dos dados cariotipicos disponiveis para Serrasalmus spilopleura. $2 \mathrm{n}=$ número diplóide; $\mathrm{M}=$ cromossomo metacêntrico; $\mathrm{SM}=$ submetacêntrico; $\mathrm{ST}=$ subtelocêntrico; $\mathrm{A}=$ acrocêntrico.

\begin{tabular}{lccccccl}
\hline BACIAS & CTÓTIPO & $2 \mathrm{~N}$ & $\mathrm{M}$ & $\mathrm{SM}$ & $\mathrm{ST}$ & $\mathrm{A}$ & REFERENCIA \\
\hline ALTO PARANÁ & "a" & 60 & 20 & 26 & 4 & 10 & Cestari \& Galetti Jr., 1992; \\
& & & & & & & Martins-Santos et al., 1994 \\
BAIXO PARANÁ & "b" & 60 & 18 & 26 & 4 & 12 & Cestari \& Galetti Jr., 1992 \\
& "c" & 60 & $18+1$ & 26 & 4 & $10+1$ & Cestari \& Galetti Jr., 1992 \\
PARAGUAI & "b" & 60 & 18 & 26 & 4 & 12 & Cestari \& Galetti Jr., 1992 \\
AMAZÔNICA & "A" & 60 & 24 & 20 & 4 & 12 & presente trabalho \\
& "B" & 60 & 24 & 26 & - & 10 & presente trabalho \\
\hline
\end{tabular}
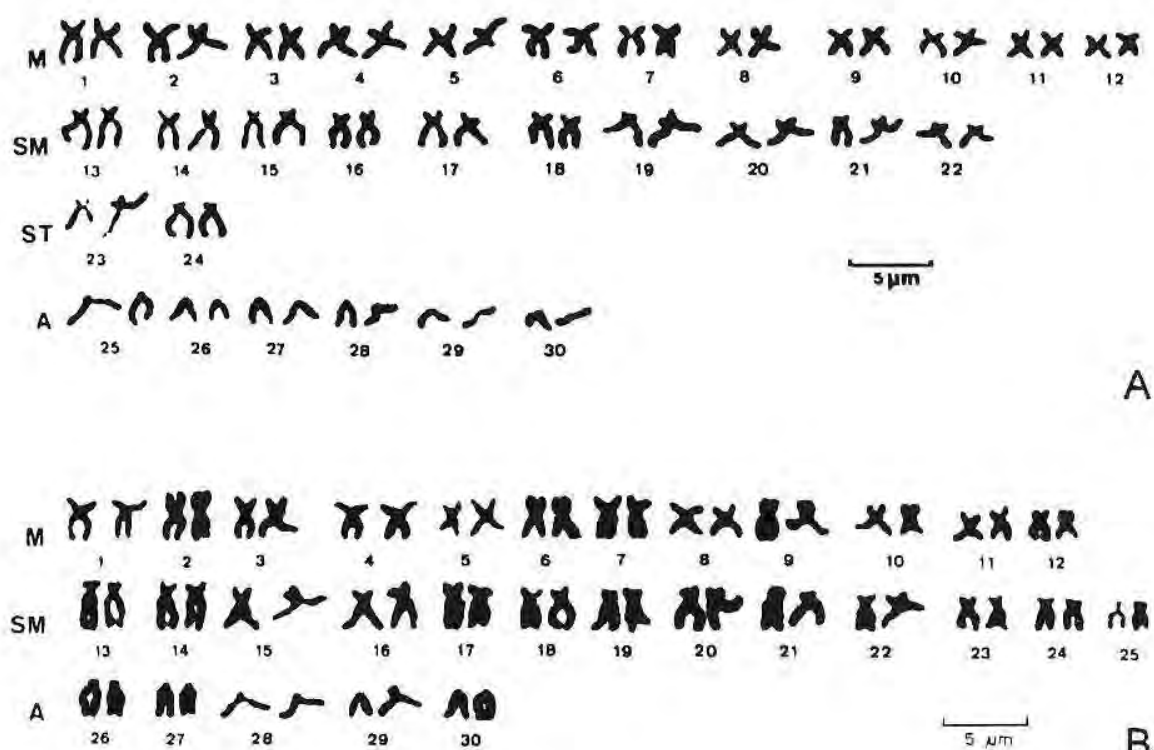

B

Figura 1. Cariótipos detectados em Serrasalmus spilopleura da Amazônia Central: A) citótipo "A", $N F=108$ e B) citótipo " $B$ ", $N F=110 . M=$ cromossomo metacêntrico; $S M=$ submetacêntrico; ST=subtelocêntrico; $A=$ acrocêntrico. 


\section{DISCUSSÃO}

Na região da Amazônia Central, casos de heteromorfismo cromossômico já foram descritos para algumas espécies de peixes como Callichtys callichtys, um siluriforme (Porto \& Feldberg, 1993); Hoplerythrinus unitaeniatus (Giuliano-Caetano \& Bertollo, 1988) e Hoplias malabaricus (Bertollo et al., 1997), dois caraciformes; e Plagioscion squamosissimus (Feldberg et al., 1999) um perciforme. Destes, o exemplo mais marcante foi aquele registrado por Giuliano-Caetano \& Bertollo (op. cit.) para H. unitaeniatus os quais encontraram em uma única região do rio Negro, quatro citótipos distintos com número de cromossomos acrocêntricos variáveis. Neste caso, as variações cromossômicas foram decorrentes de rearranjos estruturais nãoRobertsonianos, onde o número diplóide permaneceu o mesmo.

O presente trabalho evidencia pela primeira vez, a ocorrência de dois citótipos diferentes para uma espécie de piranha da Amazônia Central, $S$. spilopleura, na região de confluência dos rios Negro e Solimões, em condições de simpatria (Figs. 1A e 1B). Os citótipos, denominados A e B, distinguem-se em suas fórmulas cariotípicas, ou seja, o citótipo A apresenta 12 pares de cromossomos metacêntricos, 10 submetacêntricos, 2 subtelocêntricos e 6 acrocêntricos, enquanto o citótipo B apresenta 12 pares de cromossomos metacêntricos,
13 submetacêntricos e 5 acrocêntricos, diferenciando inclusive, no número de braços (NF), onde o citótipo A apresenta $\mathrm{NF}=108$ e o citótipo $\mathrm{B}$ $\mathrm{NF}=110$. Acredita-se que rearranjos não Robertsonianos, principalmente inversões pericêntricas devem ter ocorrido no citótipo A para dar origem ao citótipo B.

Como pode ser observado na tabela $1, S$. spilopleura tanto da Amazônia como do sistema ParanáParaguai, apresenta $2 \mathrm{n}=60$ cromossomos, mas com fórmulas cariotípicas distintas. Além disso, os citótipos do sistema Paraná-Paraguai possuem pequenos cromossomos que foram considerados diagnósticos, não observados nos citótipos amazônicos, ou seja, o citótipo "a" do alto Paraná apresenta um par de cromossomos metacêntricos pequenos; o citótipo " $\mathrm{b}$ " do baixo Paraná e Paraguai não apresenta o par de metacêntricos pequenos mas sim um par de acrocêntricos pequenos e o citótipo "c" do baixo Paraná apresenta um cromossomo do tipo metacêntrico e um do tipo acrocêntrico pequenos (Cestari \& Galetti Jr. 1992; Cestari, 1996).

Duas hipóteses foram aventadas para explicar a presença do citótipo "c" : este poderia ser decorrente de um polimorfismo cromossômico nesta espécie, próprio da região do baixo Paraná ou poderia ser o resultado de uma hibridação entre parentais pertencentes aos citótipos "a" e "b", implicando numa possível zona de hibridação na região do baixo Paraná 
(Cestari \& Galleti Jr. 1992), o que é reforçado por resultados de estudos isozímicos (Cestari, 1996).

Apesar de algumas hipóteses evolutivas já estarem disponíveis para a família Serrasalmidae (MachadoAllison, 1983; Van Every \& Kritsky, 1992; Orti et al., 1996), as relações intragenéricas nesta família ainda não estão bem definidas. O gênero Serrasalmus talvez seja o que mais careça de uma revisão taxonômica aprofundada. Assim, o fato de diferentes fórmulas cariotípicas terem sido encontradas, tanto em alopatria quanto em simpatria, podem indicar que $S$. spilopleura possivelmente não represente uma única espécie, mas sim um complexo de espécies. Neste caso, os dados citogenéticos, mostrando diferenças cromossômicas dentro e entre populações, poderão ser até mesmo decisórios nesta revisão.

\section{AGRADECIMENTOS}

Os autores agradecem aos pesquisadores: Michel Jégu (ORSTOM), pela classificação dos exemplares e Elizabeth Mendes Leão (INPA), pelas críticas e sugestões ao manuscrito. Às instituições: INPA (PPI-3370), CNPq e ORSTOM, pelo apoio financerio.

\section{Bibliografia citada}

Bertollo, L.A.C. 1986. Estimulação de mitoses em peixes. I Simp. Citogen. Evol. Aplic. Peixes Neotrop.: 30 ,

Bertollo, L.A.C.; Takahashi, C.S.; Moreira Filho, O. 1978. Cytotaxonomic considerations on Hoplias lacerdae (Pisces,
Erythrinidae) Brazil. J. Genet., 7: $103-$ 120.

Bertollo, L.A.C.; Fontes, M.S.; Fenocchio., A.S.; Cano, J. 1997. The $X_{1} X_{2} Y$ sex chromosome system in the fish Hoplias malabaricus. I. G-, C- and chromosome replication banding. Chrom. Res, 5(7): 493-499.

Cestari, M.M. 1996. Estudos citogenéticos e genéticos-bioquimicos em peixes do gênero Serrasalmus (Characiformes). Tese de doutorado. Programa de PósGraduação em Ecologia e Recursos Naturais. Universidade Federal de São Carlos, SP. 232 p.

Cestari, M.M.; Galetti Jr., P.M. 1992. Chromosome studies of Serrasalmus spilopleura (Characidae, Serrasalminae) from the Parana-Paraguay rivers: evolutionary and cytotaxonomic considerations. Copeia, 1: 108-112.

Feldberg, E.; Porto, J.I.R.; Santos, E.B.P.; Valentim, F.C.S. 1999. Cytogenetic studies of two freshwater sciaenids of the genus Plagioscion (Perciformes, Sciaenidae) from the Central Amazon. Genet. Mol. Biol., 22(3): 3451-356

Galetti Jr., P.M.; Silva, E.B.; Cerminaro, R.T. 1985. Multiple NOR system in fish Serrasalmus spilopleura (Serrasalminae, Characidae). Brazil. J. Genet., 8: 479484.

Géry, J, 1977. Characoids of the world. T.F.H. Publicacions. Neptune City N. Y. 672 p.

Géry, J.; Mahnert, V.; Dlouhy, C. 1987. Poissons characoides non Characidae du Paraguay (Pisces, Ostariophysi). Rev. Suisse Zool., 94(2): 357-464.

Giuliano-Caetano, L.; Bertollo, L.A.C. 1988. Karyotype variability in Hoplerythrinus unitaeniatus

(Characiformes, Erythrinidae) I. Chromosome polymorphism in the Rio Negro populations (Manaus state of Amazonas). Brasil. $J$. Genet., 11: 299-306.

Jégu, M. 1992. Variations du niveau marin et distribution des poissons d'eau douce en Amazonie Orientale. Collection Colloques 
et Séminaires, ed. ORSTOM: 281-297.

Jégu, M.; Santos, G.M. 1988. Le genre Serrasalmus (Pisces, Serrasalmidae) dans le bas Tocantins (Brésil, Parà), avec la description d'une espèce nouvelle, $S$. geryi, du bassin Araguaia-Tocantins. Rev. Hydrobiol. trop. 21(3):239-274.

Kner, R. 1958. Zur Familie der Characinen. II. Folge. Sitzungsber. Akad. Wiss., Wien, 32(22): 163-168.

Levan, A., Fredga, K.; Sandberg, A.A. 1964. Nomenclature for centromeric position on chromosomes. Hereditas, 52; 201-220.

Machado-Allison, A. 1983. Estudios sobre la sistemática de la subfamilia Serrasalminae (Teleostei, Characidae). Parte II. Discussion sobre la condicion monofiletica de la subfamilia. Acta Biol. Venez., 11(4): 145-195.

Martins-Santos, I.C.; Julio Jr. H.F.; Santos, S.J. 1994. Chromosome study of two species of the genus Serrasalmus (Characidae, Serrasalminae) from the Parana river. Cytologia, 59: 175-181.
Orti, G.; Petry, P.; Porto, L. I. R.; Jégu, M.; Meyer, A. 1996. Patterns of nucleotide change in mitochondrial ribosomal RNA genes and the phylogeny of piranhas. $J$. Mol. Evol., 42: 169-182.

Porto, J.I.R.; Feldberg, E. 1993. Is Callichtys Linné (Ostariophisy, Siluriformes, Callichthyidae) a monotypic genus? Acta Amaz., 23(2-3): 311-314.

Ringuelet, R.A., Aramburu, R.H.; Aramburu, A.A. 1967. Los peces argentinos de água dulce. Com. Inv. Cient., La Plata, 602p.

Santos, G.M., Jégu, M.; Merona, B. 1984. Catálogo de peixes comerciais do baixo rio Tocantins. Projeto Tucuruí. Eletronorte, INPA, Manaus. 83p.

Van Every, L.R.; Kritsky, D.C. 1992. Neotropical Monogenoidea. 18 Anacanthorus Mizelle and Price, 1965 (Dactylogyridae, Anacanthorinae) of piranha (Characoidea, Serrasalmidae) from the Central Amazon, their phylogeny, and aspects of host-parasite coevolution. J. Helminthol. Soc. Wash., 59(1): 52-75. 